\title{
A Possible Role of Intracellular Isoelectric Focusing in the Evolution of Eukaryotic Cells and Multicellular Organisms
}

\author{
Jaroslav Flegr
}

Received: 21 May 2009/ Accepted: 28 July 2009/Published online: 18 August 2009

(c) The Author(s) 2009. This article is published with open access at Springerlink.com

\begin{abstract}
A new scenario of the origin of eukaryotic cell and multicellularity is presented. A concentric $\mathrm{pH}$-gradient has been shown to exist in the cytosol of eukaryotic cells. The most probable source of such gradient is its self-formation in gradient of electric field between center and periphery of a cell. Theoretical analysis has shown that, for example, a cell of Saccharomyces cerevisiae has enough energy to continuously sustain such gradient of strength about $1.5 \mathrm{kV} / \mathrm{cm}$, the value sufficient for effective isoelectric focusing of cytoplasmic proteins. Focusing of enzymes could highly increase the effectiveness of an otherwise diffusion-limited metabolism of large cells by concentrating enzymes into small and distinct parts of a cytoplasm. By taking away an important physical constraint to the volume of cytoplasm, the intracellular isoelectric focusing enabled evolution of cells 3-4 order of magnitude larger than typical prokaryotic cells. This opened the way for the origin of phagocytosis and lately for the development of different forms of endosymbiosis, some of them resulting in an endosymbiotic origin of mitochondria and plastids. The large volume of a cell-enabled separation of nuclear and cytoplasmic compartments which was a precondition for separation of transcription and translation processes and therefore also for the origin of various RNA-preprocessing mechanisms. The possibility to regulate gene expression by postprocessing RNA and to regulate metabolism by an electrophoretic translocation enzymes between different parts of cytoplasm by changing their isoelectric points opened the way for cell and tissue
\end{abstract}

J. Flegr $(\bowtie)$

Department of Philosophy and History of Science, Charles

University, Viničná 7, 12844 Praha 2, Czech Republic

e-mail: flegr@cesnet.cz differentiation and therefore for the origin of complex multicellular organisms.

Keywords Isoelectric focusing · Cell physiology · Diffusion · Proteins - Electrophoresis - Regulation · Electric field $\cdot \mathrm{pH}$ gradient

\section{Introduction}

Two contrasting types of cell presently exist on Earth, the eukaryotic cell of Eukarya, and the prokaryotic cell of Bacteria and Archea. The presence of phospholipid membrane-bounded organelles including cell nucleus containing chromosomes with genetic information, semiautonomous organelles (mitochondria and plastids), and cytoskeleton consisting from microtubules, microfilaments, and intermediate filaments are usually considered to be major attributes of a eukaryotic cell. These characters are highly conspicuous on textbook figures drawn side-by-side in an unequal magnification; however, the most conspicuous and probably also the most important difference between real prokaryotic and eukaryotic cells is their size. A volume of typical eukaryotic cell is $3-4$ orders of magnitude larger than the volume of prokaryotic cells, excluding atypical prokaryotic forms containing a large vacuole and tiny layer of a cytoplasm. In fact, all other characters of eukaryotic cells, including the presence of semiautonomous organelles, a product of endosymbiosis, are directly or indirectly connected with the large size of eukaryotic cells. It is possible that the evolutionary invention of a mechanism enabling a primitive cell to overcome some physical barrier limiting either the size of cell or its volume to surface ratio, triggered an evolution of modern eukaryotic cell 1.5-2.7 billion years ago. 
The natural physical limit of the rate of many biochemical processes is the rate of diffusion of their reactants (Snol 1979; Koch 1996). It has been estimated that the time required for a molecule of a typical protein to move by diffusion across the interior of a HeLa cell is about 26 min (Wheatley 1985). Experimental data, however, show that fluorescently labeled proteins spread across the whole volume of a cell within seconds (Stacey and Allfrey 1977) often forming distinct spherical zones in a cytosol (Wehland and Weber 1980; Wadsworth and Sloboda 1983; Glacy 1983). The intracellular $\mathrm{pH}$ measured by NMR method with inorganic phosphate and phosphorylated intermediates of glycolysis is 7.2 and 7.0, respectively (Navon et al. 1979), which suggest that different molecules are concentrated into different regions of the cytosol (the region of maximal accumulation of glycolytic intermediates studied by NMR probably coincides with maximum of occurrence of glycolytic enzymes). The resonance peak of highly diffusible inorganic ions of inorganic phosphate is much broader than that of less diffusible molecules (Busby et al. 1978; Roberts and Jardetzky 1981). This phenomenon can be detected in the intact cell only, not in a cell homogenate. Diffusion rates of proteins, but not of polysaccharides of the same molecular weight, are much lower in the cytosol of living cells than in water of the same viscosity, and enzyme-reaction rates are often different in cell homogenates than in living cells (Bhargava 1985; Kaprelyants 1988). On the basis of these and several others facts, two groups of researches independently suggested that the eukaryotic cell uses intracellular isoelectric focusing to move and concentrate proteins and other natural ampholytes, and therefore overcomes the limitation of intracellular processes by the slow rate of diffusion in cytoplasma (Flegr 1990; Baskin et al. 2006).

Isoelectric focusing is a laboratory technique used for the separation of different proteins on the basis of their differences in isoelectric points. An isoelectric point $(\mathrm{p} I)$ is such $\mathrm{pH}$ in which an ampholyte, i.e., a molecule with both positively and negatively charged groups (zwitterion), such as a protein, has in sum a zero charge and therefore does not move in an electric field. In a mixture of ampholytes subjected to a strong enough electric field, a $\mathrm{pH}$ gradient spontaneously forms in which particular species of ampholytes (including proteins) occupy distinct zones in places with $\mathrm{pH}$ corresponding to their isoelectric point (Svensson 1961; Burggraf et al. 1995; Rilbe 1973). The presence of $\mathrm{pH}$ gradient has been proven to exist in a eukaryotic cell (Slavik 1983; Slavik and Kotyk 1984; Tsien and Poenie 1986; Paradiso et al. 1987). It is obvious that if both electric field and $\mathrm{pH}$ gradient between center and periphery exist in the interior of a cell, then all cytosolic ampholytes (e.g., proteins) must be sorted according to their pIs into concentric spherical layers, Fig. 1. It was calculated that,

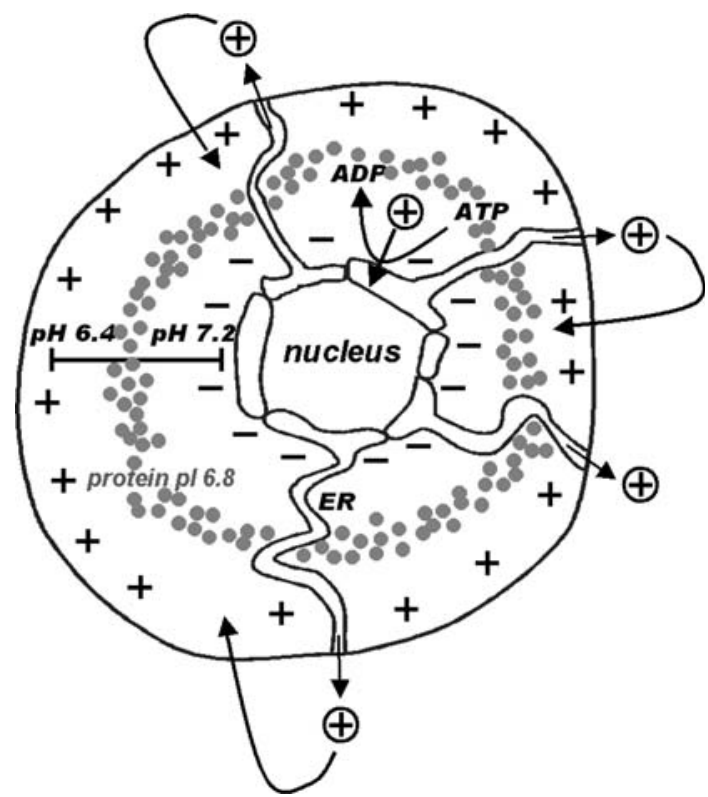

Fig. 1 Model of generation of electric field in a cell interior by the transport of protons from cell center through channels of endoplasmic reticule, and of isoelectric focusing of a protein of $\mathrm{p} I 6.8$ in selfformed $\mathrm{pH}$ gradient

for example, a cell of Saccharomyces cerevisiae has several orders of magnitude higher energetic reserves than is necessary for continuous maintenance of the intracellular difference of electric potential equal to about $0.29 \mathrm{~V}$ which represents an electric field of about $1.5 \mathrm{kV} / \mathrm{cm}$, a value more than sufficient for highly effective isoelectric focusing of cytoplasmic proteins (Flegr 1990, 1996). (It was miscalculated in the original paper that this electric field strength is only $90 \mathrm{mV} / \mathrm{cm}$, however, even such field would probably be sufficient for isoelectric focusing of intracellular proteins (Flegr 1990).) The existence of fine stratification of the cytoplasmic compartment by means of isoelectric focusing could have a great impact on the whole physiology of a cell.

In the present article, I argue that the ability of a modern cell to concentrate and move its proteins, and possibly also low-molecular weight (ampholytic) components such as aminoacids and phosphorylated metabolites, cannot only increase maximum rate and affectivity metabolic processes but can also enable fine regulation of all processes in a large cell. I suggest that the evolutionary invention of mechanisms of intracellular isoelectric focusing opened a way to the endosymbiotic origin of semiautonomous organelles through enabling an effective functioning of large cells, and therefore also to the origin of the modern eukaryotic cell. Moreover, by inventing a universal and highly evolutionarily plastic process of regulation of intracellular biochemical and physiological processes by changing isoelectric points of proteins by, e.g., phosphorylation, dephosphorylation or 
non-covalent bonding inorganic ions, and following electrophoretic translocation of modified enzyme from one place of cytoplasm to another place, evolution opened the way to fine and complex differentiation of cells and therefore to the origin of multicellular organisms.

\section{Possible Impact of Intracellular Isoelectric Focusing on Cell Physiology} Enhancement of Metabolic Reactions by Focusing
Enzymes and Their Substrates

A volume of cytoplasm of a typical eukaryotic cell is about 3-4 orders of magnitude larger than a volume of a typical prokaryotic cell. To achieve the same rate of a diffusionlimited enzyme-catalyzed reaction by increasing frequency of collisions of substrate with the enzyme, it is necessary and sufficient to concentrate both enzyme and substrate to $3-1 \%$ of the original volume of a cell. As shown in Fig. 2, such concentration can be achieved, for example, by concentrating enzyme and substrate into a spherical layer of thickness corresponding to 0.1 of the radius of the cell, about 0.3 of a cell radius from its center. It should be remembered that when two or more molecules of a substrate must interact in the same time or within a very short interval on the surface of an enzyme to give a product of

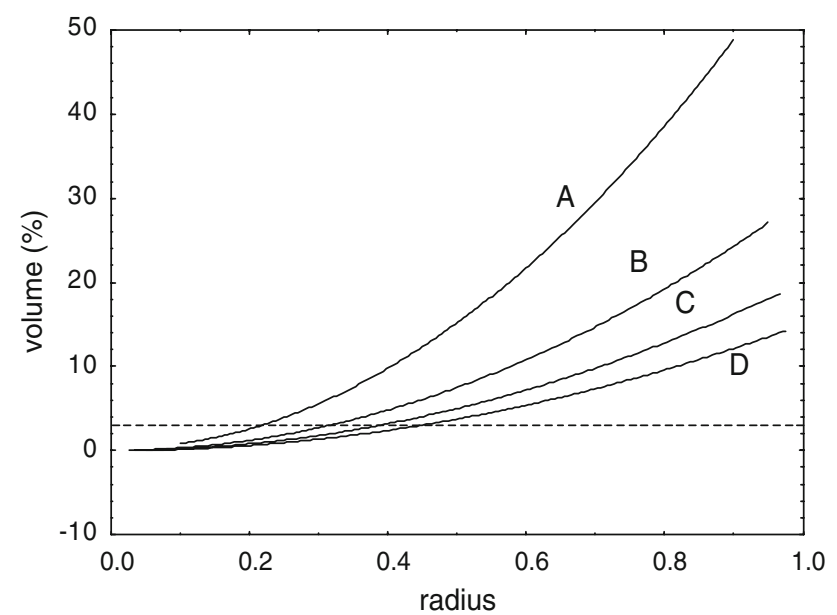

Fig. 2 Influence of thickness and position of spherical layer on its volume. The volume of the spherical layer (y-axis) is expressed in percentages of the whole volume of a sphere, lines A, B, C, and D correspond to spherical layer of thickness $1 / 5,1 / 10,1 / 15$, and 1/20 of the radius of sphere. The position of the spherical layer ( $x$-axis) is expressed as a fraction of the radius. The dashing line indicates the positions where the volume of a layer corresponds to $3 \%$ of the sphere. By focusing molecules of reactants into such a layer, a eukaryotic cell can increase the frequency of collisions of enzyme molecules with substrate and therefore increase the rate of reaction to the value observed in prokaryotic cells 3-4 orders of magnitude smaller the reaction, the enhancement of such $n$-order reaction kinetics by concentrating reactants is much higher than in the case of first and second order reaction kinetics.

The increase of rate of multienzyme metabolic pathway achieved by concentrating several (or even all) enzymes of a particular pathway into the same place of the cytoplasm is even higher. Here, the total rate of the metabolic process is limited by the ratio of backward reactions of intermediate products and forward (mostly diffusion-limited) reactions of the intermediate products with corresponding enzyme of the pathway. The concentration of enzymes participating in the same metabolic pathway in the same place can highly increase the chance that the whole multi-step process is accomplished before some of its intermediate products dissociates prematurely from its enzyme or decays due to meeting a wrong reactant or a wrong enzyme. It is probably rather common that molecules of intermediates never leave the enzyme and are instead transferred directly from one enzyme to the next by consecution of direct contacts of molecules of enzymes, by so called metabolic channeling (Cascante et al. 1994; Welch and Easterby 1994). Under such conditions, a radical increase of the rate of a metabolic process can be achieved by concentrating enzymes, without concentrating substrates. This is an important aspect; while all protein molecules are ampholytes, and pIs of most of them can probably be evolutionarily tuned to suitable values (e.g., between $\mathrm{pH} 6.4$ and 7.2 in Saccharomyces cerevisiae) to allow their focusing in any place of intracellular $\mathrm{pH}$ gradient, most low molecular weight reactants have either negative or positive charge through the whole range of cytosolic $\mathrm{pH}$ and their focusing into the cytoplasmic layer with enzyme is therefore impossible. Of course, this physical constrain can be overcome by bounding the substrate to a suitable low- or high-molecular weight carrier; however, for speeding up multienzymatic processes, focusing of low-molecular weight reactants is not necessary.

It is necessary to point out that the model of concentration of enzymes of the same metabolic pathway into the same region of a cell cannot be tested by simple comparison of their pIs. The published isoelectric points data were either collected experimentally by studying electromobility of proteins in artificial buffers and not in cytosol, or, more often, they were theoretically computed for such artificial buffers. In contrast to isoionic points $(\mathrm{pH}$ at which the overall number of negative charges equals the number of positive charges and so the molecule has no net charge in pure water), the isoelectric points ( $\mathrm{pH}$ at which the protein [or other zwitterion molecule] has zero migration in an electric field to either electrode) strongly depend on the composition of the medium. For example, an increase of the ionic strength with $\mathrm{KCl}$ from $10^{-4}$ to $10^{-2} \mathrm{M}$ shifts the isoelectric point of the collagen from $\mathrm{pH} 7.5$ to 5.3. A similar 
increase of the ionic strength in $\mathrm{CaCl}_{2}$ solutions shifts the $\mathrm{pI}$ of the same protein from 7.5 to above $\mathrm{pH} 9$ (Freudenberg et al. 2007). Actually, the isoelectric points of native proteins under in vivo conditions cannot be estimated on the basis of in vitro experiments or calculated on the basis of primary sequences of proteins. They depend not only on the aminoacid composition of a protein, its affinity to particular cations and anions present in cytosol, but also on the tertiary structure-dependent differences in rates of migration of the protein with a total negative and positive charge (it quickly fluctuates in $\mathrm{pH}$ around $\mathrm{p} I$ ) in the highly organized cytosolic matrix. The position (or colocalization) of particular proteins under in vivo condition cannot be calculated on the basis of published $\mathrm{p} I$ data.

Enhancement of Metabolic Reaction by Removal of its Product via Isoelectric Focusing

The most effective way to increase the rate of any chemical reaction is by removing its product or products. This can easily be achieved by isoelectric focusing in $\mathrm{pH}$ gradient or by any kind of electrophoretic transport when the enzymes and products of the reaction have different $\mathrm{p} I$. Many metabolic processes start with chemical modification of a molecule of substrate or its covalent or noncovalent binding to a suitable carrier. This changes the $\mathrm{p} I$ of the reactant and can cause its electrophoretic transport to a place of maximum concentration of enzymes participating in a given metabolic process, or at least enables or augments its transfer to this place by diffusion via neutralization of the electric charge of the molecule. When the last step of the metabolic process results in a change of the charge and therefore also of the $\mathrm{p} I$ of the molecule of the final product of the metabolic process, the product of the process is being continuously removed from the place where the enzymes of a particular pathway occur. It can strongly increase the rate of metabolic process by decreasing the rate of backward reaction.

\section{Enhanced Effectiveness of Metabolism via Focusing \\ Enzymes of Particular Metabolic Pathways into Different Places of a Cell}

The metabolism of a cell is an extremely complicated network of several thousands of reactions catalyzed with several thousands of enzymes. In a cell, the same molecule has a potential to participate in several anabolic and catabolic processes, i.e., the potential to be synthesized as well as the potential to be braked down in several different ways. The cell avoids the occurrence of so called futile cycles, i.e., situations when the same molecule is being synthesized by one and degraded by another pathway at the same time, by fine regulation of activity and sometimes also by the regulation of synthesis of particular enzymes. Usually, certain metabolic process, e.g., photosynthesis, is switched on while another process, e.g., photorespiration, is switched off. In the next period, the former process is switched off and the latter is switched on. Of course such regulation is never perfect, as a time delay always exists in processes of regulation. Moreover, many enzymes participating in unrelated pathways have some cross-reactivity and it is never possible or desirable to switch off all such cross-reactive enzymes. Therefore, it is not possible to avoid all futile cycles and a cell probably loses a nonnegligible part of its energetic sources in such cycles. Under specific conditions, the futile cycles can be used by cells, e.g., in thermoregulation. Under most situations, however, any mechanism that can help a cell avoid futile cycles would probably be selected for in evolution. Intracellular isoelectric focusing is just such a mechanism that can effectively prevent futile cycles. The enzymes of particular metabolic pathways can be focused into different places of the cell. Such "soft compartmentalization" of particular enzymatic pathways helps suppress futile cycles even when substrates and products are not focused and freely diffused through the cytoplasma. It is probable that the intermediate product meets the next enzyme of a particular pathway before leaving the place of high concentration of enzymes of this pathway and moving to the place of high concentration of enzymes of other pathway, where the backward metabolic process runs (Knull and Minton 1996). Using the isoelectric focusing of proteins, a cell can avoid futile cycles not only by a separation of particular metabolic processes in time (as suggested by present cytology and cell physiology) but also by a separation of metabolic processes in space (as suggested by the theory of intracellular isoelectric focusing).

When the same enzyme should participate in two or several different metabolic pathways, two or more isozyme or allozyme forms with different isoelectric points can evolve and coexist in the gene pool of the species. Isozymes or allozymes with identical specificity could be involved in different metabolic processes, depending on their $\mathrm{p} I$ and the resulting colocalization with a different set of enzymes. One isoenzyme can catalyze a predominant degradation of glucose at the place of concentration of glycolytic enzymes, while the second isozyme (with an identical specificity) can be mainly involved in a reverse reaction at the place of concentration of enzymes involved in gluconeogenesis (because of increased local concentration of a particular substrate - the product of other gluconeogenetic enzymes). This can explain why different isozyme and allozyme forms of enzymes so often differ in their isoelectric points, and why their isoelectric points so often dramatically differ from theoretically predicted mildly basic values calculated on the basis of structure of 
genetic code and model of mutation processes (Graur 1986). Therefore, the highly discriminating power of laboratory technique isoelectric focusing in allozyme analysis is not a fortuitous gift of kind Nature to molecular biologists, but rather an unavoidable result of eukaryotic cell physiology.

At least two testable predictions follow from the present model. First, existence of many isozyme forms of enzymes differing in isoelectric points from themselves and from theoretically predicted mildly basic values should be characteristic for eukaryotic, not for prokaryotic organisms without intracellular isoelectric focusing. Second, the higher viability of heterozygous individuals (with more allozymes) should be notable especially under the condition that the growth of the population is not limited by the amount of available resource, i.e., under turbidostat-like condition (Flegr 1997). When the growth of population is limited by amount of available resource, i.e., under chemostat-like conditions, the loss of resource due to futile cycles is relatively more important. Under such conditions, outbreeding can increase the heterozygosity of members of a population, and therefore the number of allozymes in their cells over optimal value. This can decrease the economy of their metabolism and therefore also their viability in comparison with more homozygous members of inbreeding populations. Organisms in turbidostatic populations, i.e., in population with predation or parasite-limited growth, should be more heterozygous and should be more sensitive to inbreeding depression, while organisms in chemostatic population, i.e., in populations with growth limited by availability of some resource, should be less heterozygous and should be more sensitive to outbreeding depression.

\section{Regulation of Cell Biochemistry and Physiology}

All biochemical and physiological processes must be finely regulated to meet the demands of the cell cycle and an external environment. Cells of multicellular organisms must also respond to the demands of the life cycle and physiology of plant, fungi, or animal organism. The regulation of intracellular processes are comprised of changes of a concentration of particular proteins including enzymes in response to changes of expression of particular genes or degradation of their RNA or protein products. Molecular biology has accumulated a huge volume of knowledge as to how internal and external signals regulate intracellular processes. However, current knowledge pertaining to how particular modifications of protein molecules, e.g., phosphorylation or dephosphorylation, result in an increase or decrease of activity of an enzyme or class of enzymes, is rather scarce. Based on analogy with known systems, it is usually supposed that chemical modification of a protein molecules results in an allosteric change in its tertiary or quaternary structure, which results in a change of its biological activity. It is true that phosphorylation or dephosphorylation of a protein could lead to change in its tertiary structure, which could result in a change of its biological properties (including its affinity to other proteins and a consequent change of biological activity of these other proteins). At the same time, the phosphorylation or dephosphorylation of a protein always leads to the change of number of charged groups and therefore to change in its isoelectric point. In an electric field and a self-formed $\mathrm{pH}$ gradient, a protein with a changed isoelectric point translocates into the new place, e.g., to the place or out of the place wherein regulated multienzymatic process runs. By moving just one of the enzymes of a particular multi-step metabolic process out of the reaction zone, the whole process can be effectively stopped.

Intracellular electrophoretically driven transport of macromolecules between different zones of a cytoplasma can be a universal mechanism that can easily evolve in response to the diversified and changeable demands of an environment, including the highly diversified demands of the tissues and organs of a multicellular organism. By changing the substrate specificity of some kinase, or by substitution of a charged aminoacids in a regulated protein, a new way of regulating an enzyme reaction can easily develop in evolution. In fact, the change of substrate specificity of an enzymatic reaction can also occur by simple change of its isoeletric point, which can occur by many different aminoacid substitutions. The substrate specificity of, e.g., kinase can remain unchanged, however, the kinase starts to phosphorylate a different set of proteins in its new place corresponding to its new isoelectric point. This gives the system of cell physiology-regulation in a eukaryotic cell an extreme evolutionary plasticity. It can be speculated that this new evolutionary plasticity played a decisive role in the evolution of sophisticated systems of tissue differentiation and therefore also in the origin of multicellular organisms.

A signal transition often includes the influx (outflux) of inorganic ions such as $\mathrm{Mg}_{2}{ }^{+}, \mathrm{K}^{+}, \mathrm{Ca}_{2}{ }^{+}, \mathrm{Cl}^{-}$from (or to) an external environment or intracellular organelle storage. Again, the concrete link between a change of concentration of inorganic ions with the change of biological properties of particular molecules and rate of particular intracellular processes is often unknown. It is clear, however, that a drastic change in concentration of inorganic ions changes the charge of many proteins by specific binding to corresponding binding sites. Depending on affinity to particular inorganic ions, different subsets of proteins respond to changes of concentration of particular inorganic ions in different ways. The number, specificity, and affinity of ion binding sites for particular inorganic ions, and therefore 
also the response of a protein to the influx/outflux of particular ions, can easily be tuned in the evolution of particular proteins.

Inorganic ions-based regulation of intracellular processes has some advantages and some disadvantages in comparison with regulation by covalent, enzyme-catalyzed modification of target molecules. The number of ions entering the cytoplasm is many orders of a magnitude higher than the number of molecules of regulating and regulated enzymes. Also, a rate of diffusion of inorganic ions is much higher than that of large molecules of proteins. Therefore, the change of charge of all molecules of regulated protein can occur nearly immediately after the change of concentration of the inorganic ion. On the other hand, transport of ions from the cytosol by molecular pump is a very ATP-consuming process. Therefore, ions-based regulation is probably used when rapid response to signal is necessary while enzymatic modification of protein, phosphorylation etc., is used when the rate of a process plays a less important role than the energetic economy of a process.

\section{Intracellular Isoelectric Focusing and the Origin of Eukaryotic Cells}

Intracellular isoelectric focusing enables efficient functioning of a large cell by passing beyond the limitation of rate of metabolic processes by rate of diffusion of molecules in cytoplasm. The possibility to build up a large cell opened a way for the evolution of new ecologic strategies, including new feeding strategies. Only a large cell can acquire nutrients by phagocytosis, by engulfment other (smaller) cells. Similarly, only a large cell can be a host of an intracellular prokaryotic parasite. Both predation by phagocytosis and endoparasitism could lead to the origin of endosymbiotic organelles-mitochondria and chloroplast (Margulis 1981). The third theoretically possible way to endosymbiosis, i.e., mutualism as suggested for example in hydrogen theory (Martin and Müler 1998), seems to be less probable as the number of systems in which the coexistence of two different organisms is useful for both symbionts is probably much lower than the number of systems in which the interaction is advantageous for one and disadvantageous for the other symbiont. Such asymmetric forms of symbiosis (as well as commensalisms) can latter evolve into mutualism as this symbiosis is probably more stable than other forms of symbioses.

A large volume of cytoplasm results in selection pressure for the origin of the cytoskeleton, which can later substitute for the exoskeleton of a prokaryotic cell-the bacterial cell wall. Again, the loss of a rigid cell wall is probably a necessary precondition for the origin of phagocytosis, and therefore for the origin of semiautonomous organelles. The origin of such cytoskeletal structures as microtubules, microfilaments, and intermediate filaments enabled not only the origin of much more complex cell morphologies (cells with cytostoma, undulating membrane, undulipodia etc.) but also much more complex cellular anatomy-systems of differentiated organelles with and without phospholipid membranes. These organelles can be either held in stable position by cytoskeleton or moved from place to place by various molecular motors attached to cytoskeleton. Microtubules and molecular motors are necessary components of mitotic and meiotic spindles, and the origin of these structures is of course critical for the emergence of two characteristic types of reproduction of modern eukaryotic cell, mitosis and meiosis. The origin of meiosis-based sexual reproduction resulted in the transition from Darwinian evolution based on competition for the highest fitness between individuals within a population, to Dawkinsonian evolution based on competition between alleles in a locus for the greatest number of their copies transmitted to the gene pool of succeeding generations (Dawkins 1976; Flegr 2008).

The origin of a nuclear envelope (composed principally from cytoskeletal components and membranous vesicles) resulted in a differentiation of a nuclear compartment. This allowed, among other things, a separation of transcription and translation processes and therefore led to the development of new strategies of genetic information processing. Namely, the separation of these two processes allowed cis and trans RNA splicing, which enabled the synthesis of many different protein products by alternative processing of RNA transcribed from the same gene (genes). Similarly, RNA editing and RNA interference-related processes enabled not only highly complex systems of regulation of gene expression and highly plastic physiological cell response to actual environmental demands (physiological plasticity of a eukaryotic organism), but also to a relatively rapid evolutionary response of regulation apparatus to environmental selection pressure (evolutionary plasticity of a eukaryotic organism).

\section{Intracellular Isoelectric Focusing and the Origin of Multicellular Organisms}

Progressive and advanced systems of regulation of gene expression by postprocessing of RNA as well as sophisticated systems of regulation of metabolism by moving molecules of enzymes between different parts of the cytoplasm by isoelectric focusing, substantially increased the capacity of a cell to differentiate into various cell types. This capacity is a necessary precondition for the development and operation of complex bodies of multicellular organisms with differentiated tissues, such as bodies of 
modern metazoa and metaphyta. It is characteristic that no representative of prokaryotic clades ever evolved into true multicellular organisms. Organismal consortia of different species of bacteria in, for example, biofilms represent an alternative way to solve complex tasks of certain environments; this solution is principally based on a loose association of different species of prokaryotic organisms with different metabolic capacities and different genetic equipment (Morris and Monier 2003; Schramm 2003; Markos et al. 2009). In the bodies of eukaryotic organisms with modern systems of regulation of gene expression and metabolism, cells equipped with the same genetic information can differentiate into forms with radically different metabolism and morphology. The existence of a common genotype and common gene pool of all cells of a multicellular organism represents an important guarantee of between-cell cooperation, namely a relatively efficient insurance against intra-organismal competition of cell lines and against different forms of selfish and ultraselfish behavior of somatic cells. A somatic cell can "delegate" the transmission of genetic information to cells of a germinal line, identical clones of itself. In large organisms, lines of somatic cells accumulate genetic differences during the development and growth of an individual due to somatic mutations and somatic recombination (Flegr 2002). In large multicellular organisms with bodies built from cells without rigid and interconnected cell walls, i.e., in the phyla of large animals, the differentiation of germinal cells must be irreversible and must occur very early to effectively prevent competition between cell lines for entering reproductive organs (Buss 1987). The existence of the Weismann barrier between somatic and germinal cell lines probably originated in evolution as an adaptation against this kind of genetic takeover in large multicellular animals.

\section{Conclusions}

In the present article, a new scenario for the origin of modern eukaryotic cell and multicellular organisms is suggested. It supposes that the first step in this process was the evolutionary invention of a molecular apparatus for intracellular focusing, namely for generation of a gradient of electric potential between the central parts and periphery of a cell. Such an electric field results in the formation of $\mathrm{pH}$ gradient in the interior of the cell, which is a necessary precondition for stratification of a cytoplasmic compartment by isoelectric focusing.

A critical point of this scenario is the existence of an electric field of sufficient strength and a $\mathrm{pH}$ gradient in the cytosol of a cell. Data from fluorescent microscopy and NMR spectroscopy suggest the existence of the $\mathrm{pH}$ gradient at least in representatives of fungi and the somatic cells of metazoa (Wehland and Weber 1980; Wadsworth and Sloboda 1983; Glacy 1983; Busby et al. 1978; Navon et al. 1979; Bhargava 1985; Kaprelyants 1988; Slavik 1983; Slavik and Kotyk 1984; Tsien and Poenie 1986; Paradiso et al. 1987). Theoretical analysis has shown that a cell has enough energy to generate an electric field of sufficient strength between its center and periphery (Flegr 1990, 1996). However, there is no direct evidence that a eukaryotic cell really generates such an electric field. The most probable mechanism of formation of the observed $\mathrm{pH}$ gradient is its self-formation in an electric field, however, two other mechanisms, namely the continuous transport of protons through cytoplasmatic or nuclear membrane, or the immobilization of molecules of ampholytes of different isoelectric points on distinct parts of a cytoskeleton, are also possible. There is some indirect evidence for the existence of isoelectric focusing in a cell (Wehland and Weber 1980; Wadsworth and Sloboda 1983; Glacy 1983; Busby et al. 1978; Navon et al. 1979; Bhargava 1985; Kaprelyants 1988; Slavik 1983; Slavik and Kotyk 1984; Tsien and Poenie 1986; Paradiso et al. 1987). However, the only direct evidence for the existence of isoelectric focusing would be an observation of distinct zones of radii corresponding to isoelectric points of fluorescently labeled proteins injected to or synthesized in cytosol of a cell. Before such direct evidence is obtained, the model of stratification of cytoplasmic compartment of eukaryotic cell by isoelectric focusing and the suggested scenarios of the origin of eukaryotic cell and origin of multicellular organisms must be considered merely hypothetical.

Acknowledgment This work was supported by Grant No. 0021620828 of the Czech Ministry of Education, Youth, and Sports.

Open Access This article is distributed under the terms of the Creative Commons Attribution Noncommercial License which permits any noncommercial use, distribution, and reproduction in any medium, provided the original author(s) and source are credited.

\section{References}

Baskin EM, Bukshpan S, Zilberstein GV (2006) pH-induced intracellular protein transport. Phys Biol 3:101-106

Bhargava PM (1985) Is the "soluble" phase of cells structured? BioSystens 18:135-139

Burggraf D, Weber G, Lottspeich F (1995) Free flow isoelectric focusing of human cellular lysates as sample preparation for protein analysis. Electrophoresis 16:1010-1015

Busby SJW, Gadian DG, Radda GK, Richards RE, Seeley PJ (1978) Phosphorus NMR-studies of compartmentation in muscle. Biochem J 170:103-114

Buss LW (1987) The evolution of individuality, 1st edn. Princeton Univ. Press, Princeton

Cascante M, Sorribas A, Canela EI (1994) Enzyme-enzyme interactions and metabolite channelling-alternative mechanisms and their evolutionary significance. Biochem J 298:313-320 
Dawkins R (1976) The selfish gene. Oxford University Press, Oxford Flegr J (1990) Does a cell perform isoelectric focusing? BioSystens 24:127-133

Flegr J (1996) Corrigendum to "Does a cell perform isoelectric focusing?" (vol 24, p 127, 1990). BioSystens 37:253

Flegr J (1997) Two distinct types of natural selection in turbidostatlike and chemostat-like ecosystems. J Theor Biol 188:121-126

Flegr J (2002) Was Lysenko (partly) right? Michurinist biology in the view of modern plant physiology and genetics. Riv Biol Biol Forum 95:259-271

Flegr J (2008) Frozen evolution or, that's not the way it is, Mr. Darwin A farewell to selfish gene. Charles University in Praque Press, Praque

Freudenberg U, Behrens SH, Welzel PB, Muller M, Grimmer M, Salchert K, Taeger T, Schmidt K, Pompe W, Werner C (2007) Electrostatic interactions modulate the conformation of collagen I. Biophys J 92:2108-2119

Glacy SD (1983) Subcellular-distribution of rhodamine-actin microinjected into living fibroblastic cells. J Cell Biol 97:1207-1213

Graur D (1986) The evolution of electrophoretic mobility of proteins. J Theor Biol 118:443-469

Kaprelyants AS (1988) Dynamic spatial-distribution of proteins in the cell. Trends Biochem Sci 13:43-46

Knull H, Minton AP (1996) Structure within eukaryotic cytoplasm and its relationship to glycolytic metabolism. Cell Biochem Funct 14:237-248

Koch AL (1996) What size should a bacterium be? A question of scale. Annu Rev Microbiol 50:317-348

Margulis L (1981) Symbiosis in cell evolution. Life and its environment on the early Earth. W.H. Freeman and Company, San Francisco

Markos A, Grygar F, Hajnal L, Kleisner K, Kratochvil Z, Neubauer Z (2009) Life as its own designer: Darwin's origin and western thought. Springer, Netherlands

Martin W, Müler M (1998) The hydrogen hypothesis for the first eukaryote. Nature 392:37-41

Morris CE, Monier JM (2003) The ecological significance of biofilm formation by plant-associated bacteria. Annu Rev Phytopathol 41:429-453

Navon G, Shulman RG, Yamane T, Eccleshall TR, Lam K-B, Baronofsky JJ, Marmur J (1979) Phosphorus $^{-31}$ nuclear magnetic resonance studies of wild-type and glycolytic pathway mutants of Saccharomyces cerevisiae. Biochemistry 18:44874499

Paradiso AM, Tsien RY, Machen TE (1987) Digital image-processing of intracellular $\mathrm{pH}$ in gastric oxyntic and chief cells. Nature 325:447-450

Rilbe H (1973) Historical and theoretical aspects of isoelectric focusing. Ann N Y Acad Sci 209:11-22

Roberts JKM, Jardetzky O (1981) Monitoring of cellular-metabolism by NMR. Biochim Biophys Acta 639:53-76

Schramm A (2003) In situ analysis of structure and activity of the nitrifying community in biofilms, aggregates, and sediments. Geomicrobiol J 20:313-333

Slavik J (1983) Intracellular $\mathrm{pH}$ topography: determination by a fluorescent probe. FEBS 156:227-230

Slavik J, Kotyk A (1984) Intracellular pH distribution and transmembrane $\mathrm{pH}$ profile of yeast cells. Biochim Biophys Acta 766:679-684

Snol SE (1979) Fiziko-khimicheskie faktory biologicheskoi evolyutsii (Physikochemical factors of biological evolution). Nauka, Moscow

Stacey DW, Allfrey VG (1977) Evidence for autophagy of microinjected proteins in HeLa-cells. J Cell Biol 75:807-817

Svensson H (1961) Isoelectric fractionation, analysis, and characterization of ampholytes in natural $\mathrm{pH}$ gradients. I. The differential equation of solute concentrations at a steady state and its solution for simple cases. Acta Chem Scand 15:325-341

Tsien RY, Poenie M (1986) Fluorescence ratio imaging-a new window into intracellular ionic signaling. Trends Biochem Sci 11:450-455

Wadsworth P, Sloboda RD (1983) Microinjection of fluorescent tubulin into dividing sea-urchin cells. J Cell Biol 97:1249-1254

Wehland J, Weber K (1980) Distribution of fluorescently labeled actin and tropomyosin after micro-injection in living tissueculture cells as observed with Tv image intensification. Exp Cell Res 127:397-408

Welch GR, Easterby JS (1994) Metabolic channeling versus free diffusion: transition-time analysis. Trends Biochem Sci 19:193197

Wheatley DN (1985) On possible importance of an intracellular circulation. Life Sci 36:299-307 